\title{
CAMERA CALIBRATION USING MULTIPLE UNORDERED COPLANAR CHESSBOARDS
}

\author{
L. Grammatikopoulos ${ }^{1, *}$, K. Adam² ${ }^{2}$ E. Petsa ${ }^{1}$, G. Karras ${ }^{2}$ \\ ${ }^{1}$ University of West Attica, GR-12243, Athens, Greece (lazaros@uniwa.gr, petsa@uniwa.gr) \\ ${ }^{2}$ National Technical University of Athens, GR-15780, Athens, Greece (adam.katerin@gmail.com, gkarras@central.ntua.gr)
}

\section{Commission II}

\begin{abstract}
KEY WORDS: Camera Calibration, Geometric Analysis, Feature Extraction, Projective Transformation, Bundle Adjustment
\end{abstract}
\begin{abstract}
The now widely available and highly popular among non-expert users, particularly in the context of UAV photogrammetry, Structurefrom-Motion (SfM) pipelines have also further renewed the interest in the issue of automatic camera calibration. The well-documented requirements for robust self-calibration cannot be always met, e.g. due to restrictions in time and cost, absence of ground control and image tilt, terrain morphology, unsuitable flight configuration etc.; hence, camera pre-calibration is frequently recommended. In this context, users often resort to flexible, user-friendly tools for camera calibration based on 2D coded patterns (primarily ordinary chessboards). Yet, the physical size of such patterns poses obvious limitations. This paper discusses the alternative of extending the size of the calibration object by using multiple unordered coplanar chessboards, which might accommodate much larger imaging distances. This is done initially by a detailed simulation to show that - in terms of geometry - this could be a viable alternative to single patterns. A first algorithmic implementation is then laid out, and results from real multi-pattern configurations, both ordered and unordered, are successfully compared. However, aspects of the proposed approach need to be further studied for its reliable practical employment.
\end{abstract}

\section{INTRODUCTION}

Since their automated pipelines may today generate high-quality spatial data at a reasonable cost, now widely available tools for 'SfM photogrammetry', particularly UAV-based, have triggered an explosion of its applications (notably in the geosciences), by attracting unprecedented numbers of users, both expert and nonexpert. This is inevitably accompanied by intensive research for reliably assessing the potential of such approaches, their limitations and the conditions for successful practices. In this context, the theoretical and practical interest in aspects of camera calibration (a crucial factor for accurate 3D reconstructions) is also renewed. Particularly so, as many users apparently tend to see in automatic camera self-calibration a guarantee for acceptable reliability and accuracy (Fraser, 2013); yet, reliance upon "black box" calibration routines is problematic since weak image block configurations will lead to imprecise and inaccurate camera parameters (Micheletti et al., 2015). Conditions for reliable selfcalibration, to avoid errors reflected as deformations in the endresults, have been well founded (James \& Robson, 2014; Luhmann et al., 2016). These generally include the need for adding oblique imagery (significant image tilt), sufficient scale variation within the images, multi-scale nadir images, an adequate number, distribution and accuracy of ground control points (GCPs), cross flight patterns (i.e. rolled images) and strong geometric features in the scene. Yet ordinary users cannot be expected to comply to similar geometric preconditions for in situ self-calibration; besides, similar image configurations are not feasible in several UAV operational situations (Eltner \& Schneider, 2015). Thus, a 'common' mapping flight, mostly consisting of similar-scale nadir images, does not represent optimal network schemes for camera parameter estimation (James et al., 2017). The problem is further aggravated with flat terrain, corridor configurations and sparse or unevenly distributed ground control (Hastedt \& Luhmann, 2015; Griffiths \& Burningham, 2018). In such cases, the obvious response is camera pre-calibration (Gerke \& Przybilla, 2016; Hastedt et al., 2016; Cramer et al., 2017). Furthermore, the requirement itself for GCPs is regarded as a serious barrier - in terms of time or cost - to a "further uptake" of UAV-based photogrammetry (Carbonneau \& Dietrich, 2016). In the absence of GCPs, pre-calibration is advised. This may also include instances of directly georeferenced photogrammetric UAV platforms (Rehak \& Skaloud, 2015; Gabrlik et al., 2018) as well as simpler applications of limited accuracy requirements (Kaiser et al., 2014) and inaccessible areas or emergency and high-risk situations (Daramola et al., 2017).

Camera pre-calibration may be carried out by independent selfcalibration, albeit subject to the above-mentioned requirements concerning camera network design, while uses of large outdoors 3D test-fields have also been made. In contrast to such more demanding approaches, plane-based approaches offer an attractive alternative; in Adam et al. (2013), for instance, the authors have reported on camera calibration based on unstructured 2D surfaces (wall graffiti). Of primary importance are of course several freely available and fully automatic tools relying on 2D patterns (and chessboards in particular) - exemplified by Bouguet's Camera Calibration Toolbox for Matlab, also included in OpenCV, and Agisoft Lens. These offer a very attractive alternative, having thus become quite popular, notably among non-expert users. The present authors have also developed such a free calibration tool, reported in Douskos et al. (2009). Chessboard patterns are easy and cheap to construct (mostly by a common printer), while distinct image corners can be robustly extracted with sub-pixel accuracy. The high demand for such flexible automatic 2D calibration tools - also used for thermal and underwater cameras (e.g. Javadnejad et al., 2019; Shortis, 2019) - is directly reflected in a steadily ongoing research which addresses issues such as computational efficiency, poor lighting/contrast, non-homogeneous illumination, overexposure, image blur, low image resolution, image noise, si-

* Corresponding author 
gnificant image distortion, missing corner points or partial occlusion of patterns, extreme imaging poses, board printing inaccuracy or deviations from planarity (recent works include Duda \& Frese, 2018; Yamaguchi et al., 2018; Yan et al., 2018; Yang et al., 2018; Hannemose et al., 2019; Meng et al., 2019; Wholfeil et al., 2019; Zhu et al., 2019). Deep learning tools have also been recently used for robust detection of checkboard corners (Donné et al., 2016; Raza et al., 2019).

Indeed, a significant number of applications of SfM/UAV photogrammetry with pre-calibrated cameras have relied on available chessboard-based calibration software (recent examples include Cucchiaro et al., 2018; Erenoğlu \& Erenoğlu, 2018; Halik \& Smaczýnski, 2018; Menge \& Lohsträter, 2018; Probst et al., 2018; Ventura et al., 2018; Wierzbicki, 2018; Burnett et al., 2019; Javadnejad et al., 2019; Kölling et al., 2019; Luppichini et al., 2019; Mahmood et al., 2019; Musci et al., 2019; Wang et al., 2019; Yurtseven, 2019), often without particular considerations, e.g. concerning focusing distance and frame coverage. Although regarded as inherently inferior to camera calibration based on 3D point arrays, 2D patterns may be expected to yield acceptable, or even comparable, accuracy for several applications by adopting powerful image block configurations (Fraser, 2013; Samper et al., 2013; Hastedt \& Luhmann, 2015; Xiang et al., 2018). Rojtberg \& Kuijper (2019) have discussed the selection of sparse views of planar patterns for optimal camera calibration concerning both pinhole camera and lens distortion parameters.

Thus, pre-calibration with a typical chessboard-based routine and camera settings identical to those of the flight has been reported to outperform self-calibrations with single-scale nadir imagery, the favored choice in environmental mapping (Griffiths \& Burningham, 2018); on the other hand, low accuracy in elevations was obtained whenever different focus settings were used for pre-calibration to ensure good frame coverage and focus (Harwin et al., 2015; Han et al., 2016; Gabrlik et al., 2018). This points to an obvious severe limitation of chessboard-based tools, namely the size of the 2D calibration object, either printed or on screen: reported sizes of such patterns do not seem to exceed $1 \times 1 \mathrm{~m}^{2}$ (Bouros et al., 2015). This physical limitation does not allow larger imaging distances or focusing at infinity, since this would inevitably produce poor frame coverage or blurred images. As a consequence, the use of a single chessboard is questionable in UAV or other outdoor applications; in this context, a new environment for camera calibration, also suitable for UAVs, is needed (Han et al., 2016; Tan et al., 2017; Lim et al., 2019).

Thus motivated, this paper is a preliminary study as to whether, and under which conditions, an extended approach based on a set of unordered coplanar chessboards might, in principle, simulate an (infeasibly large) single chessboard, to effectively accommodate longer focusing distances or wider fields of view. Multiple (non-coplanar) or 3D chessboards have already been exploited for camera-to-camera, camera-to-range sensor or multiple depth camera automatic calibration (Geiger et al., 2012; Fuersattel et al., 2017; Yin et al., 2018; Fu et al., 2019; Liu et al. 2019). Yet, to the best of our knowledge, the particular use of multiple coplanar chessboards for camera calibration has not been investigated.

Initially, the geometry of calibration adjustments with multiple coplanar chessboards was compared here, via simulated data, to that of an equivalent single-chessboard calibration. The task was to study, in an essentially geometric sense, the behavior in these two cases of the standard errors of the interior orientation parameter (IOP) values and their correlations, chiefly with the exterior orientation parameter (EOP) values. Next, the mathematical model as implemented here will be presented, and examples with real mages will be given and discussed.

\section{GEOMETRIC INVESTIGATION BY SIMULATION}

\subsection{Simulated image data}

Five identical $6 \times 7$ chessboards were assumed, with no in-plane rotations, on a planar area; four were placed symmetrically at its corners and one at its centre. This was repeated for 4 chessboard sizes $M 1-M 4$, with the dimensions of each individual board being $3.8 \%, 7.5 \%, 15 \%, 22.5 \%$, respectively, of those of the total area. These patterns were "recorded", while a single chessboard $(S)$, covering the same area outline with equal number of points (208 against 210), was too projected with identical IOPs and EOPs to provide corresponding image sets. (As an example, this configuration is roughly equivalent to an $8 \times 6.5 \mathrm{~m}^{2}$ total planar area and individual pattern sizes of $0.25 \times 0.3,0.5 \times 0.6,1.0 \times 1.2$ and $1.5 \times 1.8 \mathrm{~m}^{2}$, of which $M 2$ and $M 3$ are, of course, more realistic.)

Four 8-image sets were generated for each of the four pattern classes. The sets differed in the tilt of their camera axes (and in the respective position of the projective centres to retain similar mean image scales), i.e. in perspective deformations. The same two images of each set had roll angles of $90^{\circ}$ and $-90^{\circ}$, respectively. The four different image tilt classes (tilt 1-tilt 4 ) of Table 1 were implemented. Imaging configurations pertaining to different tilt classes and typical images are seen in Fig. 1 and Fig. 2. Although rather sub-optimal, these configurations can serve the purposes of comparison between single and multiple patterns.

\begin{tabular}{|c|c|c|c|c|}
\hline \multicolumn{5}{|c|}{ Table 1. Classes of image tilt $\left(^{\circ}\right)$} \\
\hline & tilt 1 & tilt 2 & tilt 3 & tilt 4 \\
\hline mean & 10.0 & 18.0 & 24.5 & 36.0 \\
\hline range & $1.5-17.0$ & $2.5-30.0$ & $4.5-40.0$ & $8.5-53.5$ \\
\hline
\end{tabular}

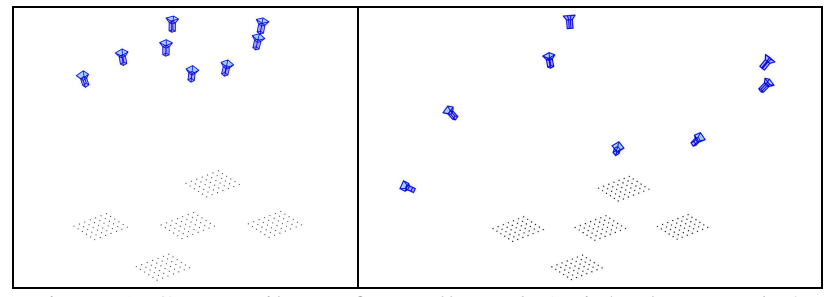

Figure 1. Camera tilts. Left: smallest tilt 1; right: largest tilt 4 .

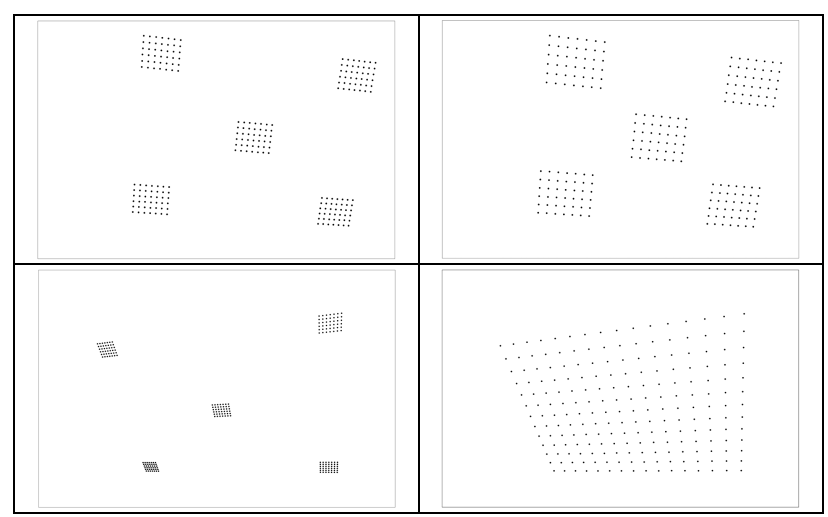

Figure 2. Above: corresponding images of small image tilt (pattern classes M3 and M4). Below: corresponding images of large image tilt (pattern classes $M 2$ and $S$ ).

\subsection{Geometric precision of parameter values}

Thus, a total of 20 self-calibrating bundle adjustments (each involving 8 images) emerged, as the combination of 4 image tilt classes with 4 pattern sizes plus the single-board case. Self-calibrating bundle adjustment included the basic 5-parameter set of IOPs: camera constant c; principal point location $\mathrm{x}_{0}, \mathrm{y}_{0}$; coeffici- 
ents $\mathrm{k}_{1}, \mathrm{k}_{2}$ of radial lens distortion. As outlined in Section 3.1, solutions with multiple patterns of course involve additional unknown parameters, i.e. the three in-plane 2D rigid transformation parameters for each but one (namely, that defining the reference system) of the patterns.

The aim here is to study the geometry (error cofactors, correlations of parameter values) of single and multiple pattern calibrations. The covariance matrix $\mathbf{C}=\mathbf{Q} \times \sigma_{0}^{2}$ of parameter values produced by an adjustment ( $\sigma_{o}$ being its standard error) represents the error propagation of random errors via the cofactor matrix $\mathbf{Q}$ (inverse matrix of normal equations); the square root of the corresponding diagonal element is the standard error of a parameter value. Thus - regardless of noise - the square root of the corresponding diagonal element of $\mathbf{Q}$ (cofactor $q_{i}$ ) directly reflects, as a propagation factor, the effect of adjustment geometry on the precision of the value of parameter $i$, which is of interest here.

2.2.1 Error cofactors of parameters: In Fig. 3 (above) the variations of cofactor values of IOPs c, xo, yo (camera constant and principal point location) with image tilt in the cases of a single $(S)$ and variably sized coplanar boards $(M)$ are presented. For $\left(\mathrm{x}_{\mathrm{o}}\right.$, $\left.\mathrm{y}_{\mathrm{o}}\right)$ the RMS value of the two cofactors is given. Corresponding $\mathrm{q}$-variations for coefficients $\mathrm{k}_{1}, \mathrm{k}_{2}$ of the radial-symmetric lens distortion polynomial are also seen in Fig. 3 (below).

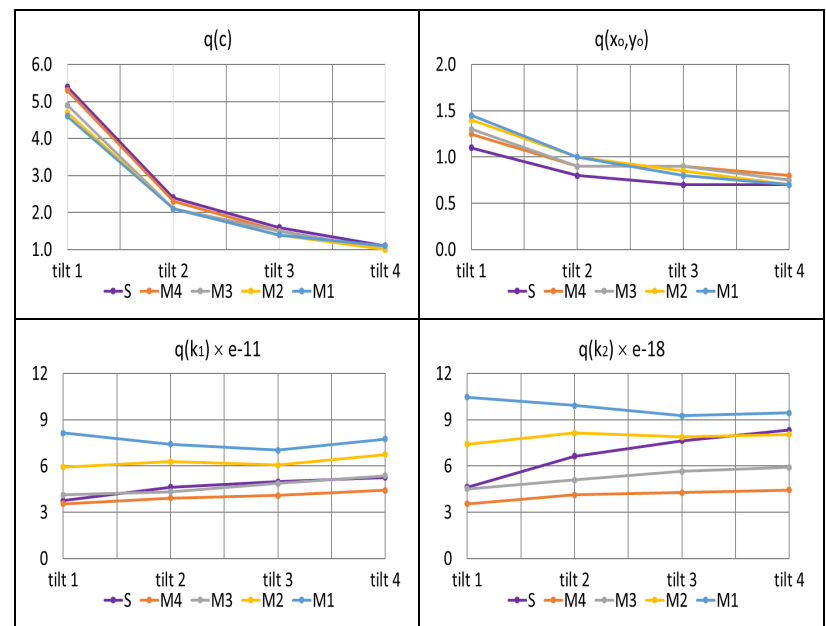

Figure 3. Cofactor $q$ values of IOP parameters according to tilt and pattern class. Above: camera constant $\mathrm{c}$ and principal point location $\left(\mathrm{x}_{0}, \mathrm{y}_{\mathrm{o}}\right)\left[\mathrm{q}\left(\mathrm{x}_{0}, \mathrm{y}_{\mathrm{o}}\right)\right.$ are RMS values of $\mathrm{q}\left(\mathrm{x}_{\mathrm{o}}\right)$ and $\left.\mathrm{q}\left(\mathrm{y}_{0}\right)\right]$. Below: coefficients $\mathrm{k}_{1}, \mathrm{k}_{2}$ of radial-symmetric lens distortion.

Image tilt expectedly strengthens the geometric precision of both $\mathrm{c}$ and $\mathrm{x}_{\mathrm{o}}, \mathrm{y}_{\mathrm{o}}$. Compared to a single 'equivalent' one, multiple patterns appear to slightly improve the precision of $\mathrm{c}$ for smaller image tilts; q-values converge with increasing image tilt. Regarding $\mathrm{x}_{\mathrm{o}}, \mathrm{y}_{\mathrm{o}}$, geometric precision is somewhat higher in the singlepattern case. For parameters k1, k2, larger pattern sizes appear as being equivalent, or slightly superior, to the single board; understandably, smaller patterns result in smaller image coverage and thus do not favour precision.

The results were validated using the single and multiple patterns (of size M2) with added Gaussian noise of different $\sigma$-levels. For each of them, 100 calibrations were performed; the RMS deviations of all IOP values for $\sigma=1$ from the correct ones were very close to the corresponding cofactor values of Fig. 3. Hence, one could provisionally claim that - from a geometric point of view at least - a (technically feasible) use of coplanar multiple patterns instead of (impracticably large) single patterns might represent a valid alternative for camera calibration for larger imaging dis- tances and, under circumstances, even provide somewhat higher precision. Intuitively, one could partly attribute this to the fact that well-distributed multiple patterns may, generally, give projective rays forming larger angles with the camera axes.

2.2.2 Correlations of parameter values: Yet, even more than standard errors (here: cofactors) of the IOP values themselves, it is their correlations with other estimated parameter values which will provide a better insight into the underlying geometry of the single and the multi-pattern scenarios. Flat chessboard-type patterns may introduce excessively high correlations between IOP and EOP values (Luhmann et al., 2016). Indeed, as estimated IOP values are here intended for autonomous (scene-independent) use as pre-calibrated data, their correlation with EOP values are of pivotal importance: weaker coupling among these parameters implies a more reliable estimation of the camera matrix. In what follows, the most significant correlations are presented (except for cases where it was indispensable, the sign of the correlation coefficient $\rho$ has been omitted to facilitate comparison). First, the correlations between $\mathrm{c}$ and the linear EOPs (i.e. image projection centre coordinates $\mathrm{X}_{\mathrm{o}}, \mathrm{Y}_{\mathrm{o}}, \mathrm{Z}_{\mathrm{o}}$ ) are shown in Fig. 4.

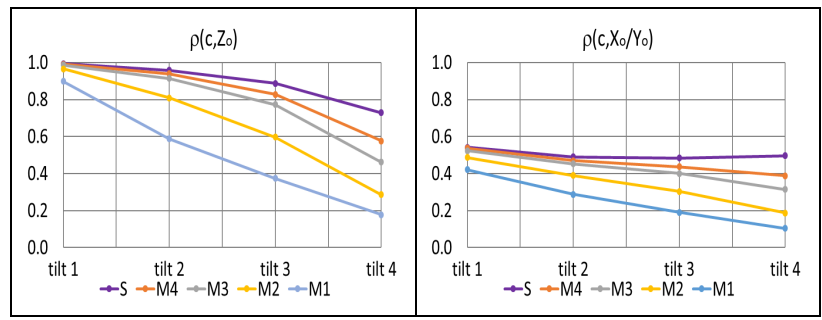

Figure 4. Correlation coefficients $(\rho)$ of camera constant $\mathrm{c}$ with EOPs $Z_{o}$ and $X_{o}, Y_{o}$. The mean absolute values of $\rho\left(c, X_{o}\right)$ and $\rho\left(c, Y_{o}\right)$ are jointly presented as $\rho\left(c, X_{o} / Y_{o}\right)$.

The c-value estimates emerge here as significantly less coupled in the multi-pattern version, notably as regards their crucial relation with the depth parameter $Z_{o}$, but also with $X_{o}, Y_{o}$. This decoupling strongly increases with tilt and decreases with pattern size. Both too large image tilts and too small patterns might, of course, be unfavourable in terms of feature extraction; hence, in practical cases suitable choices of tilt and pattern size should be made. For 'reasonable' combinations (e.g. here pattern sizes $M 2$, M3 with tilts tilt 3, tilt 4) the decrease of correlations is still evident. Correlations of $\mathrm{c}$ with the two out-of-plane image rotation angles $\omega, \varphi$ are also weakened in all cases, yet only slightly. A similar trend is observed concerning correlations of the principal point $\left(\mathrm{x}_{\mathrm{o}}, \mathrm{y}_{\mathrm{o}}\right)$ and EOPs, as illustrated in Fig. 5 regarding $\mathrm{X}_{\mathrm{o}}, \mathrm{Y}_{\mathrm{O}}$ and image roll angle $\kappa$.

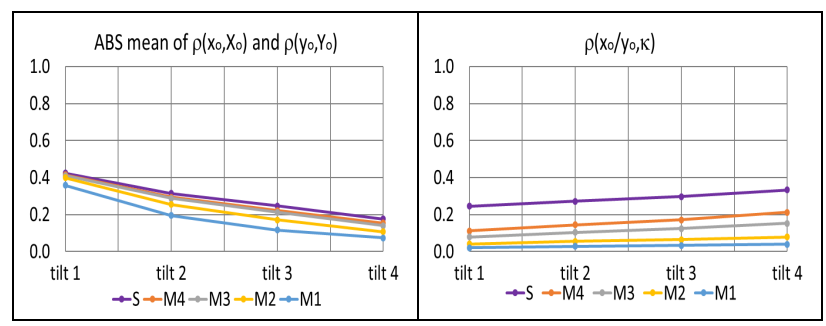

Figure 5. Left, mean absolute values of $\rho\left(\mathrm{x}_{\mathrm{o}}, \mathrm{X}_{\mathrm{o}}\right)$ and $\rho\left(\mathrm{y}_{\mathrm{o}}, \mathrm{Y}_{\mathrm{o}}\right)$; right, mean absolute values of $\rho\left(\mathrm{x}_{0}, \kappa\right)$ and $\rho\left(\mathrm{y}_{0}, \kappa\right)$.

Exception are the correlations of $\mathrm{x}_{0}, \mathrm{y}_{\mathrm{o}}$ with rotation angles $\omega$ and $\varphi$ (Fig. 6, left) which are clearly stronger in multi-pattern cases, particularly for the smaller tilts. The coupling of $x_{0}$, $y_{0}$ with the out-of-plane image angles, however, might be further dampened if more images with large roll-angles are introduced. Finally, of interest are the correlations of the radial distortion parameters $\mathrm{k}_{1}$, 
$\mathrm{k}_{2}$ with $\mathrm{Z}_{\mathrm{o}}$ (Fig. 6, right). They appear to be larger in the multipattern cases but remain relatively weak.

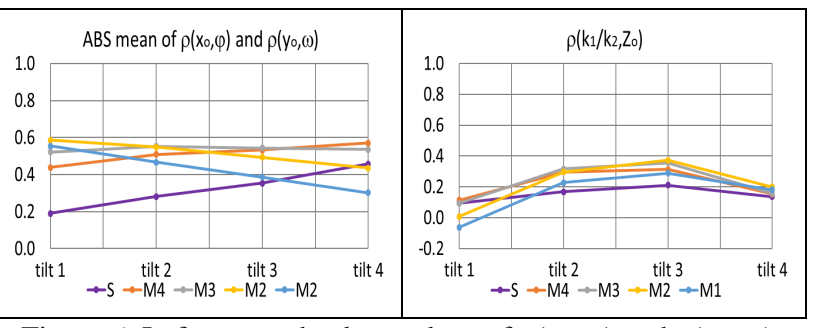

Figure 6. Left, mean absolute values of $\rho\left(\mathrm{x}_{\mathrm{o}}, \varphi\right)$ and $\rho\left(\mathrm{y}_{\mathrm{o}}, \omega\right)$; right, mean values of $\left[\rho\left(\mathrm{k}_{2}, \mathrm{Z}_{\mathrm{o}}\right)-\rho\left(\mathrm{k}_{1}, \mathrm{Z}_{\mathrm{o}}\right)\right]$.

It could be claimed that - in terms of geometry - substitution of a single by multiple coplanar chessboards appears to offer a valid option, capable of yielding equivalent, if not superior, results regarding reliable estimation of the camera matrix. This is mainly indicated by generally looser correlations among IOPs and EOPs; correlation coefficients of IOP/EOP parameter pairs $\left[\mathrm{x}_{0}, \varphi\right]$, [y, $\omega],\left[\mathrm{k}_{1}, \mathrm{Z}_{\mathrm{o}}\right],\left[\mathrm{k}_{2}, \mathrm{Z}_{\mathrm{o}}\right]$ also seem to converge for larger tilts. An explanation for this phenomenon can be found in Fig. 7 which presents the mean absolute correlations of all three 2D chessboard transformation parameters (in-plane rotation angle $\vartheta$ and translations $t_{X}, t_{Y}$ ) with all IOP and EOP values.

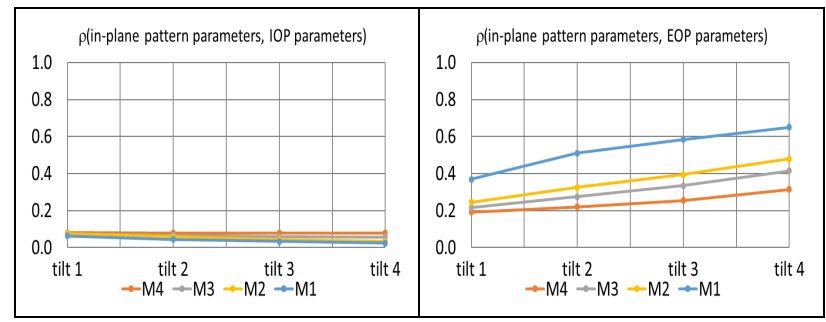

Figure 7. Means of absolute $\rho$-values of the in-plane pattern transformation parameter values with the camera IOPs (left) and EOPs (right). [It is noted that $\rho\left(\vartheta, Z_{o}\right) \sim 0$ has been ignored.]

It is seen that the additional in-plane parameters of multi-pattern cases do not actually affect IOP values but are evidently coupled with EOP estimates. This fact appears to 'reshuffle' correlations, by generally tightening them within the EOP parameter set (see examples in Fig. 8, above) and at the same time, as already noted, somewhat relaxing their correlation with IOPs. On the other hand, use of multiple patterns seems to strengthen certain correlations within the IOP set, such as those of radial distortion parameters with the camera constant and those between the principal point coordinates (Fig. 8, below). Yet, as the IOP set is intended to be further applied as a 'compact' parameter group, this represents no grave problem. The same holds true for the $\mathrm{k}_{1}, \mathrm{k}_{2}$ estimates themselves - although they were, as generally expected, very strongly intertwined with each other in all cases $\left[\rho\left(\mathrm{k}_{1}, \mathrm{k}_{2}\right) \leq-0.89\right]-$ as it has been asserted that their presence is, nevertheless, significant for modelling radial distortion (Wackrow et al., 2007).

2.2.3 Multiple chessboards on parallel planes: It is interesting to also check the effect of patterns not simply coplanar but elevated, i.e. on parallel planes. This adds a new unknown tz for each elevated pattern. Of the 5 coplanar chessboards of one size, the same two were differently elevated, in several calibration adjustments, by $5 \%-30 \%$ of the mean imaging distance. All IOP and EOP values remained the same as before. Results were assessed against those from multiple coplanar patterns. Pattern elevations improved geometric precision $\mathrm{q}(\mathrm{c})$ of the camera constant only for very small (not recommended) camera tilts, i.e. they simply seem to counterbalance the lack of significant tilt, which represents the crucial factor. The improvement of $\mathrm{x}_{0}$ and $y_{0}$ estimates is stronger (but ceases to exist for the large image tilt4); this is apparently due to correlations of $\mathrm{x}_{\mathrm{o}}$ and $\mathrm{y}_{\mathrm{o}}$ with EOPs being generally weakened by elevations. On the contrary, the distortion parameter estimates $\mathrm{k}_{1}$ and $\mathrm{k}_{2}$ from elevated patterns are generally of lower precision. Apparently, this is related to the emergence of significant correlations $(\rho=0.45-0.65)$ between $\mathrm{k}_{1}, \mathrm{k}_{2}$ and the estimated elevation values $\mathrm{t} z$ of the two patterns. Overall, these observations allow a provisional conclusion that, in principle, no notable benefits seem to be expected if replacing some coplanar patterns with elevated ones.

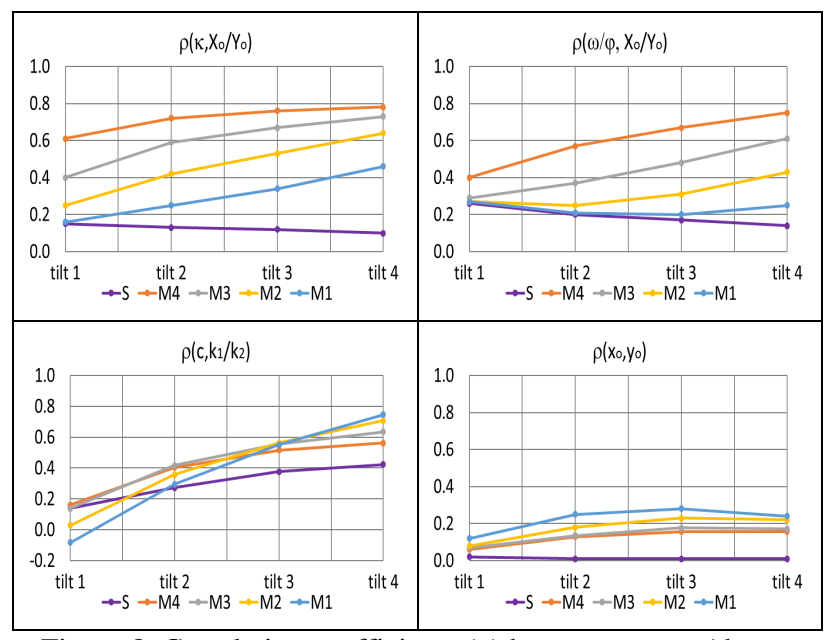

Figure 8. Correlation coefficients ( $\rho$ ) between EOPs. Above: mean absolute values of $\rho\left(\kappa, X_{o}\right)$ and $\rho\left(\kappa, Y_{o}\right)$; overall mean absolute values of $\rho$-values between $(\omega, \varphi)$ and $\left(X_{o}, Y_{o}\right)$. Below: mean values of $\left[\rho\left(k_{2}, Z_{0}\right)\right.$ and $\left.-\rho\left(k_{1}, Z_{0}\right)\right]$; mean $\rho\left(x_{0}, y_{0}\right)$ values.

2.2.4 Number of chessboards: More coplanar patterns means, of course, more points but, generally, also better distribution over the image format. The estimability of the distortion parameters, in particular, is indeed expected to benefit from the presence of image point observations in a richer variety of radial distances. Two calibration adjustments with size pattern $M 3$ were thus performed; in the second configuration four additional coplanar patterns had been added within the area described by the 5 original patterns of the first. Results are seen in Fig. 9.

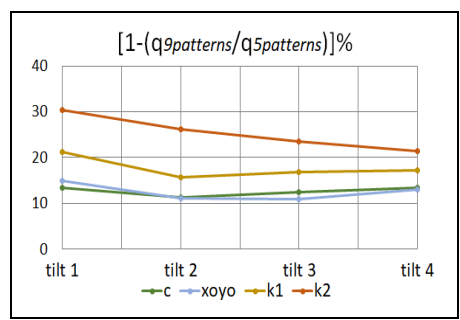

Figure 9. The effect of using 9 coplanar chessboards versus 5, presented as the proportional improvement of cofactor values q.

These results indicate that the geometry-induced improvement of the cofactor q-values is in fact the same for parameters $\mathrm{c}$ and $\mathrm{x}_{0}$, $\mathrm{y}_{\mathrm{o}}$, and might be attributed to the higher number of participating points. The markedly stronger improvement for parameters $\mathrm{k}_{1}, \mathrm{k}_{2}$, however, could be regarded also as a result of the more thorough image coverage (of the same image area) as illustrated in Fig. 10. In general, furthermore, more chessboards will allow coverage of areas close to the image corners, which is of course also crucial for reliable estimation of lens distortion. 


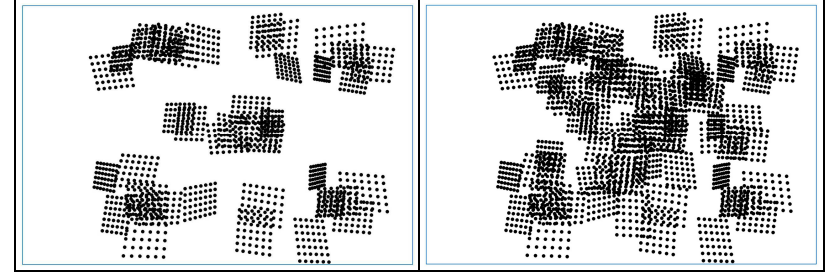

Figure 10. Overall point distribution over the whole image format (of 8 images) in the cases of 5 and 9 chessboards.

\section{CALIBRATION ALGORITHM AND APPLICATION}

\subsection{Implemented algorithm}

Our first implementation of the calibration algorithm is presented here, with the assumption that all corners of all chessboards have been extracted. Its efficiency mainly rests on the automatic identification of multiple chessboards on all available images, a process with two distinct phases: a) chessboard corner detection; b) establishment of chessboard correspondences among images.

First, in order to localize the corners of multiple chessboards appearing on several images, an algorithmic scheme based on the detectCheckerboardPoints Matlab function (which, in fact, is an implementation of the chessboard detector of Geiger et al., 2012) has been followed. Initially, a group of filter kernels selected to reveal chessboard corner patterns is convolved with the grayscale image, and thus candidate areas are extracted from the generated corner likelihood map (fusion of convolved images) by employing morphological filters (image opening and closing). The chessboard detector is then applied successively on all located image areas to allow determination of all chessboard corner coordinates with subpixel accuracy. Patterns extracted with sizes incompatible with that given by the user are ignored in the solution.

In a second stage, chessboard correspondences among images are recovered in the world plane, a consideration which drastically simplifies the complexity of this task by reducing the degrees of freedom of inter-image relation to those of a $2 \mathrm{D}$ rigid transformation. In this manner, the chessboard structure (the world plane coordinate system) is initially established by rectifying the patterns of the first image onto the world plane, based on the homography coefficients computed from an arbitrarily chosen image pattern (here, that closer to the gravity centre of all extracted chessboard corners is used as the base pattern). Similarly, all chessboards of all other images are projected recursively into the same world system. However, due to the presence of different base patterns selected in every rectification, patterns projected onto the plane from an image do not, as a rule, coincide with those from another image; in fact they differ by a 3-parameter rigid transformation ( $t_{X}, t_{Y}$ for translation, $\vartheta$ for rotation), taking into account that the non-symmetric $m \times n$ chessboard structure can be exploited. Estimation of the 3-parameter sets can, subsequently, restore pattern correspondences among images.

Although the unknown 2D rigid transformations might be found by an exhaustive search, application of a PCA (Principal Components Analysis) approach has been shown to be more efficient. Accordingly, all pattern points are first translated to their overall gravity centre and then rotated to render the axis with the higher variance horizontal. Translation of pattern points into a common coordinate system allows recovery of all pattern correspondences by matching points with the smallest Euclidean distance. Initial values for a calibration adjustment are estimated linearly by first computing the IAC (Image of the Absolute Conic), and particularly by exploiting the constraints imposed on it by homographies
H between images and the world plane (see Zhang, 2000). Camera exterior orientations are estimated via the decomposition of $\mathbf{H}$, since the camera interior orientation has been recovered.

Having thus established adequately accurate estimates for all parameters involved, the developed calibration algorithm performs the simultaneous refinement of the camera interior and exterior orientation elements for all available views, along with the exact recovery of the $2 \mathrm{D}$ translations and rotations of all other chessboard patterns with respect to the fixed reference pattern. This is carried out by minimizing image chessboard corner residuals in a least-squares bundle adjustment.

\subsection{Application and evaluation}

The configuration used here consisted of 6 coplanar $5 \times 6$ boards (1.5 cm spacing) and was recorded by two cameras: a 16 MP Sony $5 N$ and an 18 MP Canon 550D, both with fixed camera constants $\mathrm{c} \approx 20 \mathrm{~mm}$. The first dataset included 14 images, 3 of which had roll angles of $90^{\circ}$ or $-90^{\circ}$ and one $180^{\circ}$; the second consisted of 16 images, 4 of which were taken with roll angles of $90^{\circ}$ or $-90^{\circ}$. Fig. 11 presents examples of both image sets. Established pattern correspondences among images are seen in Fig. 12.

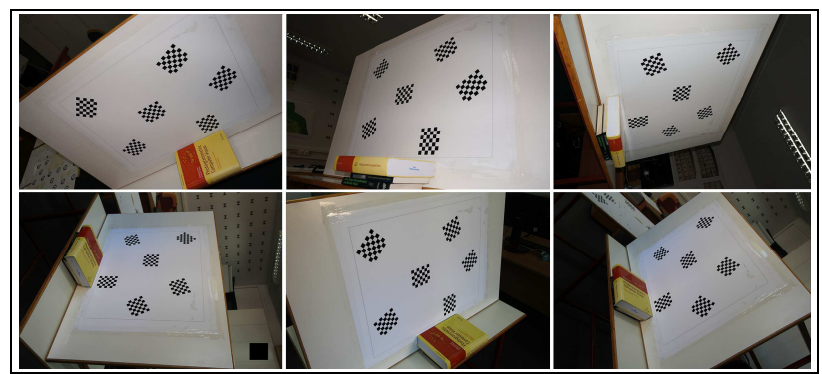

Figure 11. Images of the datasets (above: Sony; below: Canon).

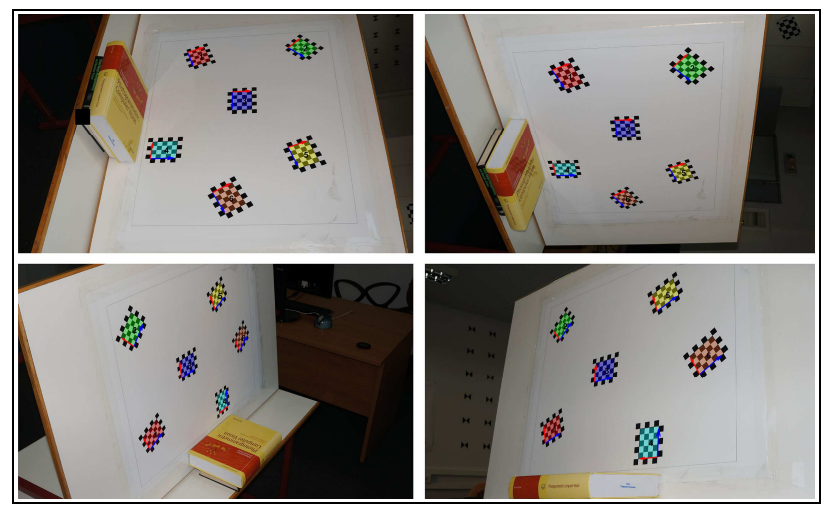

Figure 12. Details of Sony images showing all pattern correspondences established across images.

For the purposes of comparison, two calibration procedures were performed. Besides the approach described above, in the second solution the known planar coordinates of all chessboard corners participated as fixed GCPs. Thus, the studied approach of using unordered patterns could be evaluated against results from an adjustment in which all patterns were considered as fully ordered. The results of both adjustments for the two cameras are found in Tables 2 and 3 .

The results of the two approaches appear to be quite similar. The values for the camera constant differ in the two cases from their means by $0.10 \%$ and $0.23 \%$, respectively. It is also pointed out that the c-values are noticeably correlated with the distortion parameters $\mathrm{k}_{1}, \mathrm{k}_{2}$ (see Table 4); the distortion curves, however, are very close, as shown in Fig. 13. 


\begin{tabular}{|c|c|c|}
\hline \multicolumn{3}{|c|}{ Table 2. Calibration results for Sony camera (14 images) } \\
O: ordered patterns; U: unordered patterns \\
\hline \hline & $\mathrm{O}$ & $\mathrm{U}$ \\
\hline \hline$\sigma_{\mathrm{o}}(\mathrm{pixel})$ & 0.45 & 0.38 \\
\hline $\mathrm{c}($ pixel) & $4194.3 \pm 0.6$ & $4195.2 \pm 0.5$ \\
$\mathrm{X}_{\mathrm{o}}($ pixel) & $1.0 \pm 0.5$ & $-4.8 \pm 0.6$ \\
$\mathrm{y}_{\mathrm{o}}(\mathrm{pixel})$ & $22.8 \pm 0.5$ & $16.6 \pm 0.6$ \\
$\mathrm{k}_{1} \times 10^{-9}\left(\mathrm{pixel}^{-2}\right)$ & $-7.67 \pm 0.07$ & $-8.27 \pm 0.07$ \\
$\mathrm{k}_{2} \times 10^{-16}\left(\mathrm{pixel}^{-4}\right)$ & $2.87 \pm 0.23$ & $4.30 \pm 0.20$ \\
\hline \hline
\end{tabular}

\begin{tabular}{|c|c|c|}
\hline \multicolumn{3}{|c|}{$\begin{array}{c}\text { Table 3. Calibration results for Canon camera (16 images) } \\
\text { O: ordered patterns; U: unordered patterns }\end{array}$} \\
\hline \hline Canon (16 images) & $\mathrm{O}$ & $\mathrm{U}$ \\
\hline$\sigma_{\mathrm{o}}$ (pixel) & 0.40 & 0.37 \\
\hline $\mathrm{c}$ (pixel) & $4935.9 \pm 0.6$ & $4938.2 \pm 0.6$ \\
$\mathrm{X}_{\mathrm{o}}($ pixel) & $9.8 \pm 0.5$ & $8.5 \pm 0.7$ \\
$\mathrm{y}_{\mathrm{o}}($ pixel) & $19.7 \pm 0.5$ & $21.9 \pm 0.7$ \\
$\mathrm{k}_{1} \times 10^{-9}\left(\mathrm{pixel}^{-2}\right)$ & $-1.29 \pm 0.06$ & $-1.25 \pm 0.06$ \\
$\mathrm{k}_{2} \times 10^{-16}\left(\right.$ pixel $\left.^{-4}\right)$ & $3.07 \pm 0.18$ & $2.82 \pm 0.17$ \\
\hline
\end{tabular}

\begin{tabular}{|}
\begin{tabular}{|c|c|c|c|c|}
\hline Table 4. Correlation coefficients $\rho$ between $\mathrm{c}$ and $\mathrm{k}_{1}, \mathrm{k}_{2}$ \\
O: ordered patterns; U: unordered patterns \\
\hline & \multicolumn{2}{|c|}{ Sony } & \multicolumn{2}{c|}{ Canon } \\
\hline & $\mathrm{O}$ & $\mathrm{U}$ & $\mathrm{O}$ & $\mathrm{U}$ \\
\hline$\rho\left(\mathrm{c}, \mathrm{k}_{1}\right)$ & 0.44 & 0.41 & 0.27 & 0.26 \\
\hline$\rho\left(\mathrm{c}, \mathrm{k}_{2}\right)$ & -0.45 & -0.38 & -0.25 & -0.23 \\
\hline
\end{tabular}
\end{tabular}

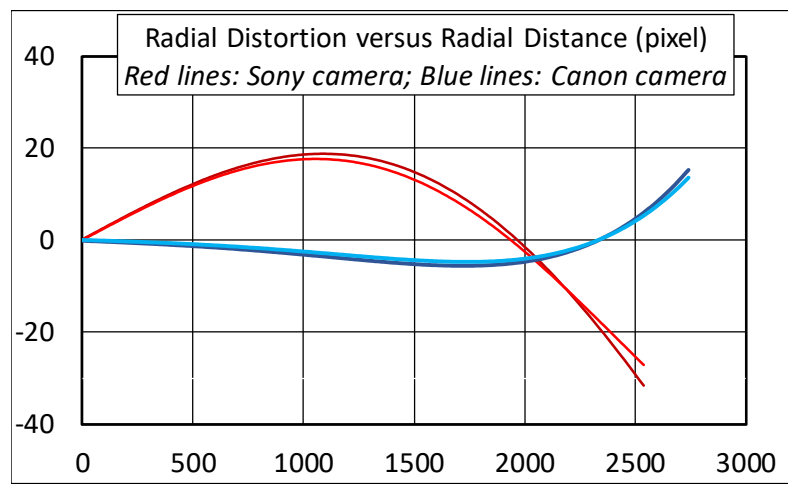

Figure 13. Calibrated radial distortion curves of the two cameras using both ordered and unordered patterns.

The principal point coordinates $\left(\mathrm{x}_{\mathrm{o}}, \mathrm{y}_{\mathrm{o}}\right)$ - generally the most sensitive camera parameters - present variations of a few pixels. This is mainly a reflection of the geometry of the adjustment: the cofactor values of $\mathrm{x}_{\mathrm{o}}, \mathrm{y}_{\mathrm{o}}$ are relatively large (namely, comparable to those of c) and, in fact, slightly higher in the case of unordered patterns. This should be considered in relation to correlations of ( $\mathrm{x}_{\mathrm{o}}, \mathrm{y}_{\mathrm{o}}$ ) with the image rotation angles which are significant. For unordered patterns, correlations of ( $\left.\mathrm{x}_{\mathrm{o}}, \mathrm{y}_{\mathrm{o}}\right)$ with the two out-ofplane rotation angles $(\omega, \varphi)$ are slightly stronger, but noticeably weaker with image roll angle $\kappa$. Similarly, correlations of $c$ with coordinates $\left(\mathrm{X}_{\mathrm{o}}, \mathrm{Y}_{\mathrm{o}}, \mathrm{Z}_{\mathrm{o}}\right)$ of the projection centre are considerably weaker, on average by $10 \%$, in the case of unordered patterns.

It may be concluded that unordered coplanar patterns appear to be, in principle, competitive with equivalent fully structured $2 \mathrm{D}$ configurations. The real positions of the patterns appear to be robustly reconstructible: the mean deviation of estimated in-plane chessboard rotation angles from their true values was $0.025^{\circ}$, while the mean deviation of estimated pattern shifts $t_{X}$, ty from their true values were, as percentage of the respective $\mathrm{X}$ and $\mathrm{Y}$ dimensions of the whole chessboard set, $0.15 \%$.

\section{CONCLUDING REMARKS}

The current popularity of widely available SfM photogrammetry tools among users in a large variety of application fields has renewed the interest in the issue of automatic camera calibration. As discussed in the introduction, the need for pre-calibrated camera geometry is not at all unusual in practical situations and is quite often tackled by resorting to the easy-to-handle automatic calibration toolboxes based on coded 2D patterns, notably of the chessboard type. Their physical limitation in size, however, has been often noted, along with their ensuing unsuitability for larger focusing distances and camera fields of view. This consideration has motivated our investigation as to whether a conventional fully structured 2D pattern might be comparably replaced by a "semistructured" configuration, such as multiple identical chessboards in unknown coplanar distributions, for the purposes of fully automatic camera calibration. Such an alternative would accommodate practicable calibration patterns of much larger size.

A detailed simulation has indicated that, in geometric terms, this seems to be, in principle at least, possible. Under the reasonable assumption that all chessboard corners have been extracted, an algorithm has been implemented, applied and evaluated. Our results proved to be comparable to those obtained when using the chessboard configuration as fully known. Obviously, further research is needed for investigating issues like chessboard number and distribution, inclusion of additional interior parameters (e.g. skewness and tangential lens distortion), pattern printing inaccuracies and, of course, for validating this approach under practical situations involving large outdoor planar test-fields.

\section{REFERENCES}

Adam K., Kalisperakis I., Grammatikopoulos L., Karras G., Petsa E., Automatic camera calibration for cultural heritage applications using unstructured planar objects. International Archives of the Photogrammetry, Remote Sensing \& Spatial Information Sciences, XL-5/W2, pp. 1-6.

Boutros N., Shortis M.R., Harvey E.S., 2015. A comparison of calibration methods and system configurations of underwater stereo-video systems for applications in marine ecology. Limnology \& Oceanography: Methods, 13:224-236.

Burnett J.D., Lemos L., Barlow D., Wing M.G., Chandler T., Torres L.G., 2019. Estimating morphometric attributes of baleen whales with photogrammetry from small UASs: a case study with blue and gray whales. Marine Mammal Science, 35(1):108- 139.

Cramer M., Przybilla H.-J., Zurhorst A., 2017. UAV cameras: overview and geometric calibration benchmark. The International Archives of the Photogrammetry, Remote Sensing \& Spatial Information Sciences, XLII-2/W6, pp. 85-92.

Cucchiaro S., Cavalli M., Vericat D., Crema S., Manel L., Alberto B., Marchi L., Cazorzi F., 2018. Monitoring topographic changes through 4D-structure-from-motion photogrammetry: application to a debris-flow channel. Environmental Earth Sciences, 77:632.

Daramola O., Olaleye J., O.G. Ajayi, Olawuni O., 2017. Assessing the geometric accuracy of UAV-based orthophotos. South African Journal of Geomatics, 6(3):395-406.

Donné S., De Vylder J., Goossens B., Philips W., 2016. MATE: Machine learning for adaptive calibration template detection. Sensors, 16, 1858. 
Douskos V., Grammatikopoulos L., Kalisperakis I., Karras G., Petsa E., 2009. FAUCCAL: an open source toolbox for fully automatic camera calibration. XXII CIPA Symposium, Kyoto, Japan.

Duda A., Frese U., 2018. Accurate detection and localization of checkerboard corners for calibration. Proc. $29^{\text {th }}$ British Machine Vision Conference (BMVC).

Eltner A., Schneider D., 2015. Analysis of different methods for $3 \mathrm{D}$ reconstruction of natural surfaces from parallel-axes UAV images. Photogrammetric Record, 30(151), pp. 279-299.

Erenoğlu R.C., Erenoğlu O., 2018. A case study on the comparison of terrestrial methods and unmanned aerial vehicle technique in landslide surveys: Sarıcaeli landslide, Çanakkale, NW Turkey. International Journal of Environment and Geoinformatics (IJEGEO), 5(3):325-336.

Fraser C.S., 2013. Automatic camera calibration in close range photogrammetry. Photogrammetric Engineering \& Remote Sensing, 79(4):381-388.

Fu B., Wang Y., Ding X., Jiao Y., Tang L., Xiong R., 2019. LiDAR camera calibration under arbitrary configurations: observability and methods. https://arxiv.org/pdf/1903.06141.pdf.

Fuersattel P., Plank C., Maier A., Riess C., 2017. Accurate laser scanner to camera calibration with application to range sensor evaluation. IPSJ Trans. on Computer Vision \& Applications, 9:21.

Gabrlik P., la Cour-Harbo A., Kalvodova P., Zalud L., Janata P., 2018. Calibration and accuracy assessment in a direct georeferencing system for UAS photogrammetry. International Journal of Remote Sensing, 39:4931-4959.

Geiger A., Moosmann F., Car Ö., Schuster B., 2012. Automatic camera and range sensor calibration using a single shot. IEEE International Conference on Robotics and Automation (ICRA), pp. 3936-3943.

Gerke M., Przybilla H.-J., 2016. Accuracy analysis of photogrammetric UAV image blocks: influence of onboard RTK-GNSS and cross flight patterns. Photogrammetrie, Fernerkundung, Geoinformation, 1/2016:17-30.

Choinowski A., Dahlke D., Ernst I., Pless S., Rettig I., 2019. Automatic calibration and co-registration for a stereo-camera system and a thermal imaging sensor using a chessboard. The International Archives of the Photogrammetry, Remote Sensing and Spatial Information Sciences, XLII-2/W13, pp. 1361-1365.

Griffiths D., Burningham H., 2018. Comparison of pre- and selfcalibrated camera calibration models for UAS-derived nadir imagery for a SfM application. Progress in Physical Geography, 43(2):215-235.

Halik Ł., Smaczýnski M., 2018. Geovisualisation of relief in a virtual reality system on the basis of low-level aerial imagery. Pure and Applied Geophysiscs, 175:3209-3221.

Han S., Park J., Lee W., 2016. On-site vs. laboratorial implementation of camera self-calibration for UAV photogrammetry. Journal of the Korean Society of Surveying, Geodesy, Photogrammetry and Cartography, 34(4): 349-356.

Hannemose M., Wilm J., Revall Frisvad J., 2019. Superaccurate camera calibration via inverse rendering. Modeling Aspects in
Optical Metrology VII, Proc. SPIE, vol. 11057, no. 11057-40.

Harwin S., Lucieer A., Osborn J., 2015. The impact of the calibration method on the accuracy of point clouds derived using unmanned aerial vehicle multi-view stereopsis. Remote Sensing, 7:11933-11953.

Hastedt H., Luhmann T., 2015. Investigations on the quality of the interior orientation and its impact in object space for UAV photogrammetry. Int. Archives of the Photogrammetry, Remote Sensing \& Spatial Information Sciences, XL-1/W4, pp. 321-328.

Hastedt H., Ekkel T., Luhmann T., 2016. Evaluation of the quality of action cameras with wide-angle lenses in UAV photogrammetry. Int. Archives of the Photogrammetry, Remote Sensing \& Spatial Information Sciences, XLI-B1, pp. 851-859.

Javadnejad F., Gillins D.T., Parrish C.E., Slocum R.K., 2019. A photogrammetric approach to fusing natural colour and thermal infrared UAS imagery in 3D point cloud generation. International Journal of Remote Sensing, 41(1):211-237.

James M.R., Robson S., 2014. Mitigating systematic error in topographic models derived from UAV and ground-based image networks. Earth Surface Processes \& Landforms, 39:1413-1420.

James M.R., Robson S., d' Oleire-Oltmanns S., Niethammer U., 2017. Optimising UAV topographic surveys processed with structure-from-motion: ground control quality, quantity and bundle adjustment. Geomorphology, 280:51-66.

Kaiser A., Neugirg F., Rock G., Müller C., Haas F., Ries J., Schmidt J., 2014. Small-scale surface reconstruction and volume calculation of soil erosion in complex Moroccan gully morphology using structure from motion. Remote Sensing, 6:7050-7080.

Kölling T., Zinner T., Mayer B., 2019. Aircraft-based stereographic reconstruction of 3D cloud geometry. Atmospheric Measurement Techniques, 12:1155-1166.

Lim P.C., Seo J., Son J., Kim T., 2019. Analysis of orientation accuracy of an UAV image according to camera calibration. The International Archives of the Photogrammetry, Remote Sensing and Spatial Information Sciences, XLII-2/W13, pp. 437-442.

Liu Z., Meng Z., Gao N., Zhang Z., 2019. Calibration of the relative orientation between multiple depth cameras based on a three-dimensional target. Sensors, 19, 3008.

Luhmann T., Fraser C., Maas H.-G., 2016. Sensor modelling and camera calibration for close-range photogrammetry. ISPRS Journal of Photogrammetry \& Remote Sensing, 115, pp. 37-46.

Luppichini M., Favalli M., Isola I., Nannipieri L., Giannecchini R., Bini M., 2019. Influence of topographic resolution and accuracy on hydraulic channel flow simulations: case study of the Versilia River (Italy). Remote Sensing, 11, 1630.

Mahmood F., Abbas K., Raza A., Khan M.A., P.W. Khan, 2019. Three dimensional agricultural land modeling using unmanned aerial system (UAS). International Journal of Advanced Computer Science and Applications, 10(1):443-449.

Menge F., Lohsträter O., 2018. Einbindung von UAV-Messungen in bestehende Prozesse der Markscheiderei der MIBRAG. $19^{\text {ter }}$ Geokinematischer Tag des Institutes für Markscheidewesen und Geodäsie, Freiberg, pp. 138-146. 
Meng C., Wang Q., Wu L., Guan S., Wu Y. Wang T., 2019. A fast X-corner detection method based on block-search strategy. Advances in Mechanical Engineering, 11(3):1-11.

Micheletti N., Chandler J.H., Lane S., 2015. Structure from Motion (SfM) Photogrammetry. Geomorphological Techniques, 2.2, British Society of Geomorphology.

Musci M.A., Aicardi I., Dabove P., Lingua A.M., 2019. Reliability of the geometric calibration of a hyperspectral frame camera. The Int. Archives of the Photogrammetry, Remote Sensing \& Spatial Information Sciences, XLII-2/W13, pp. 1701-1707.

Probst A., Gatziolis D., Strigul N., 2018. Intercomparison of photogrammetry software for three-dimensional vegetation modelling. Royal Society Open Science, 5, 172192.

Raza S.N., Rehman H.R., Lee S.G., Choi G.S., 2019. Artificial intelligence based camera calibration. $15^{\text {th }}$ IEEE International Wireless Communications \& Mobile Computing Conference (IWCMC), pp. 1564-1569.

Rehak M., Skaloud J., 2015. Fixed-wing micro aerial vehicle for accurate corridor mapping. ISPRS Annals of Photogrammetry, Remote Sensing \& Spatial Information Sciences, II-1/W1, pp. 23-31.

Rojtberg P., Kuijper A., 2019. Efficient pose selection for interactive camera calibration. arXiv:1907.04096v1.

Samper D., Santolaria J., Brosed F.J., Majarena A.C., Aguilar J.J., 2013. Analysis of Tsai calibration method using two and threedimensional calibration objects. Machine Vision \& Applications, 24:117-131.

Shortis M., 2019. Camera calibration techniques for accurate measurement underwater. $3 D$ Recording and Interpretation for Maritime Archaeology, Coastal Research Library, 31:11-27.

Tan L., Wang Y., Yu H., Zhu J., 2017. Automatic camera calibration using active displays of a virtual pattern. Sensors, 17, 685.

Ventura D., Bonifazi A., Gravina M.F., Belluscio A., Ardizzone G., 2018. Mapping and classification of ecologically sensitive marine habitats using unmanned aerial vehicle (UAV) imagery and object-based image analysis (OBIA). Remote Sensing, 10, 1331.

Wackrow R., Chandler J.H., Bryan P., 2007. Geometric consistency and stability of consumer-grade digital cameras for accurate spatial measurement. Photogrammetric Record, 22(118): 121 -134 .

Wang S., Baum A., Zarco-Tejada P.J., Dam-Hansen C., Thorseth A., Bauer-Gottwein P., Bandini F., Garcia M., 2019. Unmanned aerial system multispectral mapping for low and variable solar irradiance conditions: potential of tensor decomposition. ISPRS J. of Photogrammetry \& Remote Sensing, 155:58-71.

Wierzbicki D., 2018. Multi-camera imaging system for UAV photogrammetry. Sensors, 18, 2433.

Wohlfeil J., Grießbach D., Ernst I., Baumbach D., Dahlke D., 2019. Automatic camera system calibration with a chessboard enabling full image coverage. The International Archives of the Photogrammetry, Remote Sensing \& Spatial Information Sciences, XLII-2/W13, pp. 1715-1722.

Xiang T.-Z., Xia G.-S., Zhang L., 2018. Mini-UAV-based remote sensing: techniques, applications and prospectives. https://arxiv.org/pdf/1812.07770.pdf.

Yamaguchi M., Truong T.P., Mori S., Nozick V., Saito H., Yachida S., Sato H., 2018. Superimposing thermal-infrared data on 3D structure reconstructed by RGB visual odometry. IEICE Transactions on Information and Systems, E101(5):1296-1307.

Yan Y., Yang P., Yan L., Wan J., Sun Y., Tansey K., Asundi A., Zhao H., 2018. Automatic checkerboard detection for camera calibration using self-correlation. Journal of Electronic Imaging, 27(3), no. 033014.

Yang T., Zhao Q., Wang X., Zhou Q., 2018. Sub-pixel chessboard corner localization for camera calibration and pose estimation. Applied Sciences, 8, 2118.

Yin L., Wang X., Ni Y., Zhou K., Zhang J., 2018. Extrinsic parameters calibration method of cameras with non-overlapping fields of view in airborne remote sensing. Remote Sensing, 10, 1298.

Yurtseven H., 2019. Comparison of GNSS-, TLS- and different altitude UAV-generated datasets on the basis of spatial differences. ISPRS International Journal of Geo-Information, 8, 175.

Zhang Z., 2000. A flexible new technique for camera calibration. IEEE Transactions on Pattern Analysis \& Machine Intelligence, 22(11):1330-1334.

Zhu Z., Wang X., Liu Q., Zhang F., 2019. Camera calibration method based on optimal polarization angle. Optics and Lasers in Engineering, 112:28-135. 